\title{
Inducción de resistencia en frijol contra conchuela Epilachna varivestis con nutrición y homeopatía
}

\author{
Cesáreo Rodríguez Hernández ${ }^{1}$ - Felipe de Jesús Ruiz Espinoza ${ }^{2}$ - Luis Manuel Serrano Covarrubias ${ }^{3}$ \\ - Ildefonso Ronquillo Cedillo ${ }^{4}$
}

Universidad Intercultural del Estado de México, México

Recepción: 27/06/2017

Aceptación: 03/08/2017

\section{Resumen}

El control de insectos plaga con insecticidas organosintéticos provoca varios problemas, por ello se han implementado varias alternativas; sin embargo, estas no se han integrado para lograr un mejor manejo. Esta investigación se realizó para evaluar el efecto de la fertilización mineral u orgánica y sin fertilizar, suelo convencional u orgánico y, con o sin agronosodes homeopáticas en el desarrollo de la conchuela del frijol y el daño foliar del adulto.

Para ello se sembró frijol con estos tratamientos y se ofreció como planta u hoja cortada a diez larvas de primer instar en dos experimentos. Se evaluaron diversos parámetros en la biología del insecto y el daño foliar diario del adulto. La composta a una y tres toneladas $\mathrm{ha}^{-1} \mathrm{y}$ el suelo orgánico, por separado, reducen de 7 a 14.6\% la duración larval, respecto a la fertilización mineral y al suelo convencional; y a dosis de 1.8 a $3.6 \mathrm{t} \mathrm{ha}^{-1}$ y el agronosode a la $200 \mathrm{CH}$ reducen en $50 \%$ o más el daño foliar del adulto, pero combinados pierden el efecto. El suelo orgánico combinado con agronosode a la $200 \mathrm{CH}$ reduce en 85.7\% la viabilidad pupal. La integración de suelo orgánico con composta 3 t ha-1 de composta o con agronosode a la $200 \mathrm{CH}$ perjudica al insecto y protege al cultivo.

Palabras clave: agronosode, abono orgánico, daño, fertilizante mineral, frijol, Epilachna varivestis.

\begin{abstract}
IThe pest insect control with organosynthetic pesticides provoke many problems; for this reason, many alternatives have been implemented. However, these ones have not been included to reach a better management. This research was conducted to evaluate the effect of the mineral fertilization or organic without fertilizing it, conventional soil or organic and with or without agronosodes homeopathic in the development of the bean's shell and also the foliage damage of the old one.
\end{abstract}

To this effect, bean was planted with these treatments and it was offered as plant or cut leaf to ten maggots of first instar in two experiments. The different parameters in the insect biology and the daily foliage damage of the old one were evaluated. The compost at one and three tons ha ${ }^{-1}$, and the organic soil, independently, reduce from 7 to $14.6 \%$ the larval duration regards to the mineral fertilization and to the conventional soil and doses of 1.8 to $3.6 \mathrm{tha}^{-1}$ and the agronosode at $200 \mathrm{CH}$ reduce in $50 \%$ or more the adult foliage damage, but combined lose the effect. The organic combine soil with agronosode at $200 \mathrm{CH}$ reduces in $85.7 \%$ the pupal viability. The organic soil integration with compost $3 \mathrm{t}$ $\mathrm{ha}^{-1}$ of compost or with agronosode at $200 \mathrm{CH}$ prejudices the insect and protects the crop.

Key words: agronosode, organic compose, damage, mineral fertilizer, bean, Epilachna varivestis.

1. Doctor en Entomología, Profesor-Investigador, Colegio de Postgraduados, Departamento de Entomología y Acarología, México; email: clandero@colpos.mx

2. Doctor en Ciencias Agrarias, Profesor-Investigador, Centro Regional Universitario Anáhuac, Universidad Autónoma Chapingo, México; email: agrohomeopatia@hotmail.com

3. Doctor en Sistemas de Producción, Coordinador Académico, Centro Regional Universitario Centro Norte, México; email: frijol_uach@msn.com 4. Máster en Ciencias Agrícolas, Profesor-Investigador, Universidad Intercultural del Estado de México, México; email: ildefonso.ronquillo@uiem.edu.mx 


\section{Introducción}

En México, el frijol es uno de los cultivos más importantes, tanto por la producción total como por la superficie sembrada que se cultiva en todos los estados geográficos. Sin embargo, hay varios factores que reducen la producción, entre ellos las plagas. La principal plaga, en zonas productoras de clima templado, es la conchuela del frijol (Epilachna varivestis), la cual se controla comúnmente con la aspersión de insecticidas organosintéticos en detrimento de la salud, del ambiente y de enemigos naturales; razón por la que se buscan alternativas.

Entre algunas de ellas han destacado la fertilización orgánica y la agrohomeopatía. La fertilización orgánica, a través de abonos, disminuye el daño de la tortuguilla rayada del pepino (Acalymma vittatum), de la diabrótica de once puntos (Diabrotica undecimpunctata), del gusano del cuerno (Manduca quinquemaculata) (Yardim et al., 2006); de la catarinita de la papa (Leptinotarsa decemlineata) (Boiteau, Lynch y Martin, 2008), y del chapulín (Hieroglyphus nigrorepletus) (Dixit, Dixit y Samson 2009). Además, reduce la tasa de crecimiento de la mariposita blanca de la col (Pieris rapae crucivora) (Hsu, Shen y Hwang 2009), y el peso y la supervivencia de la palo- milla dorso de diamante (Plutella xylostella) (Johnson et al., 2012).

La agrohomeopatía ${ }^{5}$, a través del uso de los homeopáticos de la propia plaga (agronosodes), ha mostrado diversos efectos; reduce en 14\% la población de ninfas del pulgón ceniciento del manzano (Dysaphis plantaginea), con la 6CH (Wyss et al., 2010); disminuye en $81.76 \%$ la actividad de forrajeo de la hormiga defoliadora (Atta sexdens piriventris), a la 30CH (Giesel et al., 2013); y reduce en 83$100 \%$ la emergencia de adultos del gorgojo del frijol (Acanthoscelides obtectus) en la primera generación, a la $30 \mathrm{CH}^{6}$ (Deboni, 2009). No obstante, no se han combinado ambas alternativas, por esta razón se buscó determinar el efecto de la fertilización mineral u orgánica y sin fertilizar, suelo convencional u orgánico y sin o con agronosodes, solos y en combinación en la duración larval, pupal y larva-adulto, la viabilidad larval y pupal y, el peso de pupa y adulto, así como en el daño del adulto de la conchuela del frijol E. varivestis.

\section{Materiales y Métodos}

Esta investigación se realizó en condiciones de invernadero en las instalaciones de Agricultura Alternativa Orgánica y Sustentable México (AALTERMEX), en Texcoco, Estado

5. Agrohomeopatía es la aplicación de sustancias vegetales, animales y minerales en dosis infinitesimales, potenciadas (dinamizadas) a través de un proceso de sucusión (una agitación, que consiste en golpear el frasco de arriba hacia abajo, sobre una superficie resistente y elástica).

6. La letra $\mathrm{C}$ hace referencia a diluciones centesimales y la letra $\mathrm{H}$ se refiere a la escala hanemaniana, en honor a Samuel Hahnemann, el iniciador de la homeopatía. Para preparar la CH se usa una parte de la sustancia por cada 99 partes de líquido (Ruiz y Rodríguez, 2013). 
de México, y en el área de Insecticidas Vegetales (IV) del Campus Montecillo (CM), Colegio de Postgraduados (CP) en Ciencias Agrícolas durante los meses de agosto del año 2013 a junio del año $2014^{7}$.

La cría de conchuela del frijol E. varivestis existente en la área de IV, CM, CP se incrementó en todos los estados biológicos, lo cual consistió en tomar masas de huevos y colocarlas en cajas Petri, con algodón humedecido, para evitar su deshidratación y permitir la eclosión; una vez que emergieron las larvas, se colocaron en plantas de frijol variedad flor de mayo con al menos las primeras hojas verdaderas, y se confinaron en jaulas en las que se alimentaron cotidianamente, hasta terminar su desarrollo.

Cuando las larvas se transformaron en pupas, éstas se colectaron y se pusieron en cajas Petri para su desarrollo, y cuando los adultos emergieron se colocaron en la jaula de adultos, donde se les proporcionó continuamente alimento, consistente en plantas de frijol flor de mayo con primeras hojas verdaderas, donde además copularon y ovipositaron. Los huevos se colectaron diariamente y se pusieron en cajas Petri para su eclosión. Así se consiguió tener alta población de los cuatro estados biológicos en todas las edades, y disponer de larvas de primer instar para los experimentos de esta investigación.

Se sembró frijol de la variedad flor de mayo en macetas de $8 \mathrm{~L}$ con diferente fertilización mineral (90-40-20, 40-20-10 y 20-10-0, usando como fuentes: urea, superfosfato de calcio triple y cloruro de potasio) u orgánica $(0.1$, $1.0,3.0$ y $4.5 \mathrm{t} \mathrm{ha}^{-1}$ con composta ${ }^{8}$ proporcionada por AALTERMEX) o sin fertilizar; suelo convencional, obtenido de una parcela experimental en el CM, CP, u orgánico, proporcionado por AALTERMEX; y sin o con aplicación vía riego del agronosode de la conchuela a la 10 y $200 \mathrm{CH}$, proporcionados por el Dr. Felipe de Jesús Ruiz Espinoza, responsable del área de Agrohomeopatía del Centro Regional Universitario del Anáhuac de la Universidad Autónoma Chapingo, Texcoco, Estado de México, el cual fue elaborado a partir de los cuatro estados biológicos de la conchuela y con follaje dañado por ésta. En el primer experimento se utilizó suelo de una parcela en descanso, en tanto que en el segundo, éste fue tanto convencional como orgánico. La elección de los tratamientos en ambos casos se efectuó mediante una matriz baconiana a la que se le agregaron algunas interacciones, conformando catorce combinaciones.

7. Los autores también se referirán a estos lugares por las abreviaciones entre paréntesis.

8. La composta fue elaborada con materiales secos (rastrojo de maíz y pajas de cereales), hierbas verdes, y tierra de cultivo en proporción 4:2:1 en volumen, a una relación C:N inicial de 25-35:1. 
A los treinta días se colocaron diez larvas de primer instar de conchuela del frijol E. $v a$ rivestis en el haz de las hojas, confinadas en bolsas de tul en el primer experimento o cortadas como en el segundo experimento, donde se colocaron en un vaso transparente con el peciolo dentro de un gotero con agua para mantener la turgencia; y se cambiaron a discreción. A medida que se fue desarrollando la larva, se fue alimentando de plantas de hasta setenta días de edad, en ambos experimentos.

Al formarse las pupas se determinó la duración larval (DL), y la viabilidad larval (VL), y un día después se colectaron y pesaron en una balanza analítica para obtener el peso de pupa (PP); posteriormente se colocaron en cajas Petri en condiciones de laboratorio para que continuaran su desarrollo. Cuando emergieron los adultos se registró la duración pupal (DP) y se calculó la viabilidad pupal (VP); un día después de su formación, se pesaron en una balanza analítica para obtener el peso de adulto (PA); y por último, con la suma de todos los tiempos, se determinó la duración larva-adulto (DLA) ${ }^{9}$.

Con los adultos obtenidos en el primer experimento se realizó una prueba de daño que consistió en colocar una hoja trifoliolada de frijol, de la misma planta donde se desarrollaron sus estados inmaduros, en un gotero dentro de un vaso transparente en el que se introdujeron dos adultos de conchuela por cada repetición. Transcurridas veinticuatro horas se retiraron y se colectaron las hojas, las cuales se escanearon para determinar el área dañada, utilizando el software de manejo de imágenes de licencia libre IMAGEJ versión 1.48 (Rasband, 2014).

El diseño experimental en los tres ensayos realizados fue un completamente al azar con catorce tratamientos (que variaron de acuerdo al experimento) y cuatro repeticiones. A los datos de la biología de la conchuela y de daño foliar del adulto se les realizaron las pruebas de Bartlett y Shapiro Wilk para verificar los supuestos de homogeneidad de varianzas y normalidad de los errores, respectivamente. Cuando éstos se cumplieron, como en el daño foliar del adulto, se realizó un análisis de varianza; y en caso de diferencias significativas entre tratamientos se realizó la prueba de Tukey ( $\alpha \leq 0.05)$, y cuando no se cumplieron los supuestos, como en la biología de la conchuela en ambos experimentos, se utilizó el método propuesto por Conover e Iman (1981): se transformaron los datos originales a rangos y se realizó el análisis de varianza para ver la diferencia entre tratamientos, y cuando ésta se presentó se realizó la prueba de Tukey $(\alpha \leq 0.05)$.

Para comparar grupos de tratamientos se realizaron pruebas de contrastes en las que se compararon fertilización mineral contra orgánica, y sin o con agronosodes $(\alpha \leq 0.05)$.

9. Los autores también se referirán a estas fases mediante las abreviaciones entre paréntesis. 
Con los datos del primer experimento, en fertilización orgánica se realizaron regresiones para determinar el efecto de ésta en la biología y en el daño del adulto, para esto se usó el método de Stepwise. Todos los análisis se realizaron con el paquete estadístico SAS 9.1 (SAS Institute, 2004).

\section{Resultados y Discusión}

En el primer experimento, en el que se evaluaron la fertilización y los agronosodes sin considerar el factor suelo, se observó (Tabla 1) que no existe efecto en los siete parámetros evaluados en la biología de la conchuela respecto al tratamiento sin fertilización ni agronosodes. Sin embargo, en el análisis de contrastes, al comparar los tratamientos de composta con los tratamientos de fertilizante mineral, se observó una reducción significativa $(\mathrm{P} \leq 0.036 \mathrm{y}$ 0.030) de DL y DLA que queda evidenciada en la composta 1 y $3 \mathrm{t} \mathrm{ha}^{-1}$, respecto a la fertilización mineral 40-20-10; sin dejar de considerar que los contrastes analizaron grupos de tratamientos, ya que el agronosode $200 \mathrm{CH}$ mostró un efecto en DLA que no se logró evidenciar en los contrastes, (ver tabla 1).

La reducción de la duración larval también la observaron Boiteau et al. (2008) en la larva de la catarinita de la papa (L. decemlineata), con la aplicación de altas dosis de abono orgánico respecto a la aplicación de fertilizantes minerales; pero el abono también puede incrementar la duración larval, como en la mariposita blanca de la col $P$. rapae crucivora
(Hsu et al., 2009) y en la palomilla dorso de diamante (P. xylostella) (Johnson et al., 2012), aunque no a dosis altas. Tal reducción, aunque en teoría puede parecer beneficiosa para el insecto al tener más generaciones por ciclo, en el trabajo de Boiteau et al. (2008) se relaciona con factores adversos a la plaga como menor duración de las etapas en las que provoca el daño (aspecto no evaluado en esta investigación); ya que a la par de la reducción de la duración larval hubo menor consumo de follaje, por lo que se puede considerar que la reducción de la duración larval es una consecuencia de una mala alimentación por parte de la larva.

En lo que respecta al daño diario del adulto en función de la fertilización, éste varió de $0.8 \mathrm{~cm}^{2}$ en composta $3 \mathrm{t} \mathrm{ha}^{-1}$ (sin fertilización mineral ni agronosode) a $3.6 \mathrm{~cm}^{2}$ en fertilizante mineral 40-20-10 ( in composta ni agronosode) (Tabla 2), con diferencias estadísticamente significativas entre los tratamientos de composta a 1 y $3 \mathrm{t} \mathrm{ha}^{-1}$ contra los de fertilización mineral media y baja y el tratamiento sin fertilizar. Estos datos muestran que cuando se usa composta se reduce el daño del adulto si se aplican dosis cercanas a $3 \mathrm{t} \mathrm{ha}^{-1}$, pudiendo ser mayor a 50\% con la aplicación de 1.8 a $3.6 \mathrm{t}$ $\mathrm{ha}^{-1}$, o menor al daño medio en las dosis que se alejan de éstas hacia 0 o $4.5 \mathrm{t} \mathrm{ha}^{-1}$, porque la relación entre aplicación de composta y el daño diario del adulto de conchuela se explica por una ecuación de tercer grado, de acuerdo al análisis de regresión (Figura 1). 
Tabla 1. Duración de los estados inmaduros (DL, DP y DLA), viabilidad (VL y VP) y pesos (PP y PA) en conchuela alimentada de frijol cultivado con diferente fertilización y agronosode

\begin{tabular}{|c|c|c|c|c|c|c|c|c|c|}
\hline \multicolumn{2}{|c|}{ Fertilizante } & \multirow[b]{2}{*}{ Agronosode } & \multirow[b]{2}{*}{$\begin{array}{c}\text { DL } \\
\text { (d) } \\
\text { rango* }\end{array}$} & \multirow[b]{2}{*}{$\begin{array}{c}\text { VL } \\
\begin{array}{c}\text { (porcentaje) } \\
\text { rango }\end{array}\end{array}$} & \multirow[b]{2}{*}{$\begin{array}{c}\text { PP } \\
\text { (mg) } \\
\text { rango }\end{array}$} & \multirow[b]{2}{*}{$\begin{array}{l}\text { DP } \\
\text { (d) } \\
\text { rango }\end{array}$} & \multirow[b]{2}{*}{$\begin{array}{c}\text { VP } \\
\begin{array}{c}\text { (porcentaje) } \\
\text { rango }\end{array}\end{array}$} & \multirow[b]{2}{*}{$\begin{array}{c}\text { PA } \\
\text { (mg) } \\
\text { rango }\end{array}$} & \multirow[b]{2}{*}{$\begin{array}{l}\text { DLA } \\
\text { (d) } \\
\text { rango }\end{array}$} \\
\hline $\begin{array}{l}\text { Mineral } \\
\text { (NPK) }\end{array}$ & $\begin{array}{l}\text { Composta } \\
\left(\mathrm{t} \mathrm{ha}^{-1}\right)\end{array}$ & & & & & & & & \\
\hline $40-20-10$ & 0.0 & No & $\begin{array}{l}(29.6) \\
38.4 \quad \mathrm{a} \\
\end{array}$ & (41.7) $13.3 \mathrm{a}$ & $\begin{array}{l}(41.8) \\
27.8 \mathrm{a}\end{array}$ & $\begin{array}{c}(6.9) \\
30.1 \mathrm{ab}\end{array}$ & (100) $30 \mathrm{a}$ & $\begin{array}{l}(31.8) \\
21.0 \mathrm{a}\end{array}$ & $\begin{array}{l}(36.5) \\
39.1 \quad \mathrm{a}\end{array}$ \\
\hline $90-40-20$ & 0.0 & No & $\begin{array}{r}(29.0) \\
29.3 \mathrm{ab}\end{array}$ & (70.8) $34.6 \mathrm{a}$ & $\begin{array}{l}(40.7) \\
18.0 \mathrm{a}\end{array}$ & $\begin{array}{c}(6.7) \\
22.5 \mathrm{ab}\end{array}$ & (100) $30 \mathrm{a}$ & $\begin{array}{l}(32.7) \\
22.5 \mathrm{a}\end{array}$ & $\begin{array}{r}(35.7) \\
29.4 \mathrm{ab}\end{array}$ \\
\hline $20-10-00$ & 0.0 & No & $\begin{array}{r}(28.5) \\
29.3 \mathrm{ab} \\
\end{array}$ & $(55.6) 22.6 \mathrm{a}$ & $\begin{array}{l}(42.0) \\
25.4 \mathrm{a}\end{array}$ & $\begin{array}{c}(7.0) \\
36.5 \text { a }\end{array}$ & (100) $30 \mathrm{a}$ & $\begin{array}{l}(31.8) \\
20.0 \mathrm{a}\end{array}$ & $\begin{array}{l}(35.5) \\
31.3 \mathrm{ab}\end{array}$ \\
\hline 00-00-00 & 4.5 & No & $\begin{array}{l}(28.1) \\
21.0 \mathrm{ab}\end{array}$ & (88.9) $47.5 \mathrm{a}$ & $\begin{array}{l}(42.3) \\
31.0 \mathrm{a}\end{array}$ & $\begin{array}{c}(6.9) \\
27.5 \mathrm{ab}\end{array}$ & (100) $30 \mathrm{a}$ & $\begin{array}{l}(33.9) \\
30.8 \mathrm{a}\end{array}$ & $\begin{array}{r}(34.9) \\
21.5 \mathrm{ab}\end{array}$ \\
\hline 00-00-00 & 3.0 & No & $\begin{array}{l}(28.4) \\
19.6 \mathrm{~b}\end{array}$ & $(80.6) 41.7 \mathrm{a}$ & $\begin{array}{l}(41.9) \\
23.8 \mathrm{a}\end{array}$ & $\begin{array}{c}(6.7) \\
25.3 \mathrm{ab}\end{array}$ & (96.4) $23.8 \mathrm{a}$ & $\begin{array}{l}(33.1) \\
26.3 \mathrm{a}\end{array}$ & $\begin{array}{r}(35.1) \\
20.1 \mathrm{ab}\end{array}$ \\
\hline 00-00-00 & 1.0 & No & $\begin{array}{l}(27.5) \\
15.0 \quad b\end{array}$ & (55. 6) $23.0 \mathrm{a}$ & $\begin{array}{l}(44.3) \\
36.5 \mathrm{a}\end{array}$ & $\begin{array}{c}(6.9) \\
33.5 \mathrm{ab}\end{array}$ & (87.5) $22.8 \mathrm{a}$ & $\begin{array}{l}(34.0) \\
30.6 \mathrm{a}\end{array}$ & $\begin{array}{l}(34.4) \\
13.6 \mathrm{~b}\end{array}$ \\
\hline 00-00-00 & 0.1 & No & $\begin{array}{l}(29.5) \\
32.3 \mathrm{ab}\end{array}$ & (55.6) $23.9 \mathrm{a}$ & $\begin{array}{l}(39.2) \\
22.5 \mathrm{a}\end{array}$ & $\begin{array}{c}(6.5) \\
15.8 \mathrm{~b}\end{array}$ & (95.0) $23.4 \mathrm{a}$ & $\begin{array}{l}(32.6) \\
24.8 \mathrm{a}\end{array}$ & $\begin{array}{r}(36.0) \\
29.5 \mathrm{ab}\end{array}$ \\
\hline 00-00-00 & 0.0 & No & $\begin{array}{r}(29.5) \\
32.5 \mathrm{ab} \\
\end{array}$ & (48.6) $18.4 \mathrm{a}$ & $\begin{array}{l}(41.9) \\
27.9 \mathrm{a}\end{array}$ & $\begin{array}{c}(6.7) \\
23.3 \mathrm{ab}\end{array}$ & (91.7) $23 \mathrm{a}$ & $\begin{array}{l}(33.8) \\
32.3 \mathrm{a}\end{array}$ & $\begin{array}{l}(36.2) \\
34.0 \mathrm{ab}\end{array}$ \\
\hline $40-20-10$ & 0.0 & $10 \mathrm{CH}$ & $\begin{array}{c}(28.4) \\
28.1 \mathrm{ab}\end{array}$ & (72.2) $35.0 \mathrm{a}$ & $\begin{array}{l}(43.6) \\
38.0 \mathrm{a}\end{array}$ & $\begin{array}{c}(6.8) \\
32.4 \mathrm{ab}\end{array}$ & (95) $23.4 \mathrm{a}$ & $\begin{array}{l}(33.9) \\
38.0 \mathrm{a}\end{array}$ & $\begin{array}{r}(35.2) \\
27.9 \mathrm{ab}\end{array}$ \\
\hline $40-20-10$ & 0.0 & $200 \mathrm{CH}$ & $\begin{array}{l}(29.3) \\
36.0 \quad \mathrm{a}\end{array}$ & (61.1) $28.6 \mathrm{a}$ & $\begin{array}{l}(41.0) \\
25.3 \mathrm{a}\end{array}$ & $\begin{array}{c}(6.9) \\
31.5 \mathrm{ab}\end{array}$ & (100) $30 \mathrm{a}$ & $\begin{array}{l}(32.9) \\
25.8 \mathrm{a}\end{array}$ & $\begin{array}{l}(36.2) \\
38.4 \quad \mathrm{a}\end{array}$ \\
\hline 00-00-00 & 0.0 & $200 \mathrm{CH}$ & $\begin{array}{c}(28.3) \\
26.6 \mathrm{ab}\end{array}$ & (56.9) $25.4 \mathrm{a}$ & $\begin{array}{l}(41.1) \\
24.5 \mathrm{a} \\
\end{array}$ & $\begin{array}{c}(6.7) \\
23.8 \mathrm{ab}\end{array}$ & (100) $30 \mathrm{a}$ & $\begin{array}{l}(31.6) \\
28.9 \mathrm{a}\end{array}$ & $\begin{array}{r}(35.0) \\
22.3 \mathrm{ab} \\
\end{array}$ \\
\hline $40-20-10$ & 3.0 & No & $\begin{array}{r}(28.7) \\
27.3 \mathrm{ab}\end{array}$ & (66. 7) $30.4 \mathrm{a}$ & $\begin{array}{l}(41.6) \\
29.3 \mathrm{a}\end{array}$ & $\begin{array}{c}(6.8) \\
24.0 \mathrm{ab}\end{array}$ & (100) $30 \mathrm{a}$ & $\begin{array}{l}(32.9) \\
22.9 \mathrm{a}\end{array}$ & $\begin{array}{r}(35.5) \\
27.5 \mathrm{ab}\end{array}$ \\
\hline $40-20-10$ & 3.0 & $200 \mathrm{CH}$ & $\begin{array}{r}(27.5) \\
12.3 \quad \mathrm{~b} \\
\end{array}$ & (58.3) $27.4 \mathrm{a}$ & $\begin{array}{l}(41.6) \\
25.8 \mathrm{a}\end{array}$ & $\begin{array}{c}(7.0) \\
36.5 \mathrm{a}\end{array}$ & (100) $30 \mathrm{a}$ & $\begin{array}{l}(33.1) \\
27.9 \mathrm{a}\end{array}$ & $\begin{array}{l}(34.5) \\
13.8 \quad b\end{array}$ \\
\hline 00-00-00 & 3.0 & $200 \mathrm{CH}$ & $\begin{array}{c}(28.8) \\
34.3 \mathrm{ab}\end{array}$ & (52.8) $23.3 \mathrm{a}$ & $\begin{array}{l}(43.0) \\
31.3 \mathrm{a}\end{array}$ & $\begin{array}{c}(6.7) \\
22.5 \mathrm{ab}\end{array}$ & (100) $30 \mathrm{a}$ & $\begin{array}{l}(34.3) \\
35.1 \mathrm{a}\end{array}$ & $\begin{array}{r}(35.5) \\
33.8 \mathrm{ab}\end{array}$ \\
\hline
\end{tabular}

* Medias de rangos con letras similares en columnas no son diferentes estadísticamente de acuerdo con la prueba de Tukey $(\alpha \leq 0.05)$. 
Tabla 2. Daño diario del adulto de conchuela alimentado con hojas cortadas de plantas de frijol

\begin{tabular}{|c|c|c|c|}
\hline \multicolumn{2}{|c|}{ Fertilizante } & \multirow[b]{2}{*}{ Agronosode } & \multirow[b]{2}{*}{$\begin{array}{c}\text { Daño } \\
\left(\mathrm{cm}^{2} \text { adulto }^{-1} \mathrm{~d}^{-1}\right)\end{array}$} \\
\hline Mineral (NPK) & Composta $\left(\mathrm{t} \mathrm{ha}^{-1}\right)$ & & \\
\hline $40-20-10$ & 0.0 & No & $3.6 \mathrm{a}$ \\
\hline $90-40-20$ & 0.0 & No & $2.9 \mathrm{ab}$ \\
\hline 20-10-00 & 0.0 & No & $3.5 \mathrm{a}$ \\
\hline 00-00-00 & 4.5 & No & $3.4 \mathrm{ab}$ \\
\hline 00-00-00 & 3.0 & No & $0.8 \mathrm{c}$ \\
\hline 00-00-00 & 1.0 & No & $2.4 \mathrm{~b}$ \\
\hline 00-00-00 & 0.1 & No & $3.4 \mathrm{ab}$ \\
\hline 00-00-00 & 0.0 & No & $3.1 \mathrm{ab}$ \\
\hline $40-20-10$ & 0.0 & $10 \mathrm{CH}$ & $3.3 \mathrm{ab}$ \\
\hline $40-20-10$ & 0.0 & $200 \mathrm{CH}$ & $1.9 \mathrm{bc}$ \\
\hline 00-00-00 & 0.0 & $200 \mathrm{CH}$ & $2.8 \mathrm{ab}$ \\
\hline $40-20-10$ & 3.0 & No & $2.4 \mathrm{~b}$ \\
\hline $40-20-10$ & 3.0 & $200 \mathrm{CH}$ & $2.3 \mathrm{bc}$ \\
\hline 0 & 3.0 & $200 \mathrm{CH}$ & $3.1 \mathrm{ab}$ \\
\hline
\end{tabular}

* Medias con letras similares no son diferentes estadísticamente de acuerdo con pruebas de Tukey $(\alpha \leq 0.05)$.

En el caso de los homeopáticos, la aplicación del agronosode a la 200CH mostró un efecto significativo: reducción del daño en 50\% respecto al fertilizante mineral, aunque no mostró diferencias significativas con el tratamiento sin fertilizar. El agronosode a $10 \mathrm{CH}$ no fue diferente del testigo.

La fertilización como lo hacen los productores (40-20-10) fue la que obtuvo el mayor daño por la conchuela, pero cuando se apli- có composta $3 \mathrm{t} \mathrm{ha}^{-1}$ o agronosode $200 \mathrm{CH}$ se modificó este efecto, provocando reducción del daño foliar de 33 y 47\% respectivamente, lo cual puede ser una opción para los productores que usan fertilizante mineral, y que sin dejar esta opción pueden añadir de 1.8 a 3.6t ha ${ }^{-1}$ de composta o aplicar el agronosode $200 \mathrm{CH}$ para disminuir significativamente la defoliación por adultos de conchuela. El uso de composta con agronosode a la $200 \mathrm{CH}$ no reduce el daño. 


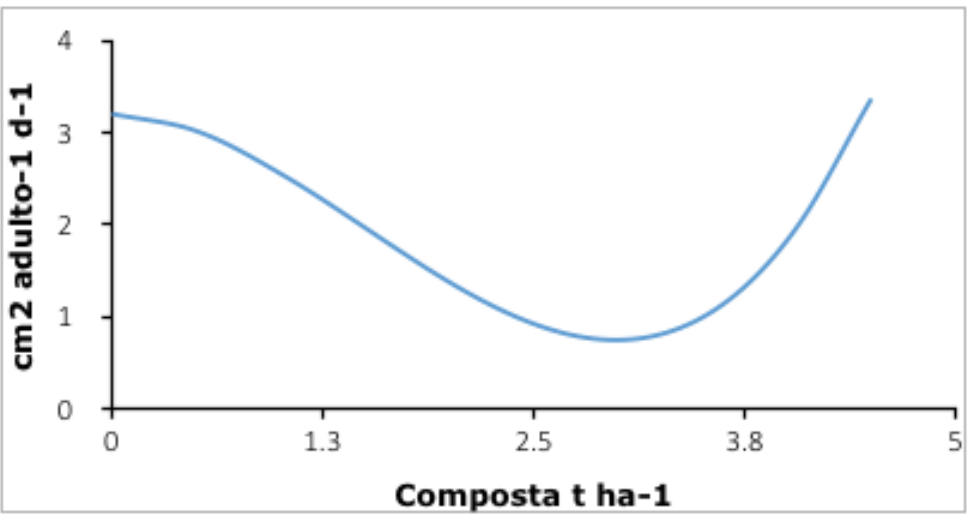

Daño $=3.20-0.84$ composta $^{2}+0.19$ composta $^{3}$ $\mathrm{R}^{2}=0.70$

Figura 1. Daño diario del adulto de conchuela en función de la aplicación de composta.

La reducción en más del 50\% del daño foliar con la aplicación de composta a dosis de 1.8 a $3.6 \mathrm{t} \mathrm{ha} \mathrm{a}^{-1}$ ya se había observado en tortuguilla rayada del pepino (A. vittatum), diabrótica de once puntos (D. undecimpunctata) y gusano del cuerno (M. quinquemaculata) (Yardim et al., 2006), y en chapulín (H. nigrorepletus) (Dixit et al., 2009); así como en la catarinita de la papa (L. decemlineata), donde se redujo de dos a cinco veces el consumo y el tiempo de alimentación, en plantas con abono orgánico respecto a las que se les aplicó fertilizante mineral (Boiteau et al., 2008).

De manera general, acorde a este primer experimento, el uso de la composta reduce el tiempo de desarrollo de la conchuela del frijol y su daño foliar en comparación con la aplicación de fertilizante mineral; y el agronosode a la $200 \mathrm{CH}$ reduce en $50 \%$ el daño del adulto de conchuela respecto a las plantas con fertilización mineral. Circunstancias similares de reducción de tiempo de desarrollo larval y disminución de consumo, se observaron en la catarinita de la papa (L. decemlineata) con el uso de abonos orgánicos (Boiteau et al., 2008); y en el caso de los homeopáticos se puede reducir hasta en $81.76 \%$ el daño, como lo reportan Giesel et al. (2012 y 2013) con el forrajeo de las hormigas defoliadoras Acromyrmex sp. y A. sexdens piriventris con el agronosode a la $30 \mathrm{CH}$. Este efecto de reducción del daño foliar del adulto es importante ya que; aunque no se evaluó, se puede pensar que si los adultos consumen menos follaje tendrán reducción en la fertilidad y fecundidad, y habrá por ello también menor oviposicion como se observó en el barrenador europeo del maíz $O$. nubilalis (Phelan et al., 1995 y 1996).

En el segundo experimento no hubo significancia de los catorce tratamientos en los parámetros de DP, VP, PA y DLA (Tabla 3). No obstante, en suelo orgánico con agronosode a 
la $200 \mathrm{CH}$ y $\sin$ fertilización se obtuvo $14.3 \%$ de VP; dato que no se analizó estadísticamente por ser bajo. En este tratamiento, de las pupas que se formaron en las repeticiones, una fue viable y generó un adulto que emergió 1.5 días después de lo normal con peso aproximado de una tercera parte de lo normal. En esta población, lo que comió como larva le afectó en su desarrollo posterior, afectando en $85.7 \%$ el paso de pupa a adulto.

Tabla 3. Duración de los estados inmaduros (DL, DP y DLA), viabilidad (VL y VP) y pesos (PP y PA) en conchuela alimentada con hojas cortadas de frijol cultivado con diferente fertilización, suelo y agronosode

\begin{tabular}{|c|c|c|c|c|c|c|c|c|c|c|}
\hline \multicolumn{2}{|c|}{ Fertilizante } & \multirow[b]{2}{*}{ Suelo } & \multirow[b]{2}{*}{$\begin{array}{l}\text { Agrono- } \\
\text { sode }\end{array}$} & \multirow[b]{2}{*}{$\begin{array}{c}\text { DL } \\
(d) \\
\text { rango }^{*}\end{array}$} & \multirow[b]{2}{*}{$\begin{array}{c}\text { VL } \\
\text { (por- } \\
\text { centaje) } \\
\text { rango }\end{array}$} & \multirow[b]{2}{*}{$\begin{array}{c}\text { PP } \\
\text { (mg) } \\
\text { rango }\end{array}$} & \multirow[b]{2}{*}{$\begin{array}{c}\text { DP } \\
\text { (d) } \\
\text { rango }\end{array}$} & \multirow[b]{2}{*}{$\begin{array}{c}\text { VP } \\
\text { (por- } \\
\text { centaje) } \\
\text { rango }\end{array}$} & \multirow[b]{2}{*}{$\begin{array}{c}\text { PA } \\
\text { (mg) } \\
\text { rango }\end{array}$} & \multirow[b]{2}{*}{$\begin{array}{c}\text { DLA } \\
\text { (d) } \\
\text { rango }\end{array}$} \\
\hline $\begin{array}{l}\text { Mine- } \\
\text { ral } \\
(\mathrm{NPK})\end{array}$ & $\begin{array}{l}\text { Com- } \\
\text { posta } \\
\left(\mathrm{t} \mathrm{ha}^{-1}\right)\end{array}$ & & & & & & & & & \\
\hline $\begin{array}{c}90-40- \\
20\end{array}$ & 0.0 & Conv. & Agua & $\begin{array}{l}(29.6) \\
27.8 \mathrm{a}\end{array}$ & $\begin{array}{c}(50.0) \\
26.4 \mathrm{ab}\end{array}$ & $\begin{array}{l}(30.5) \\
21.4 \mathrm{~b}\end{array}$ & $\begin{array}{c}(5.4) \\
12.9 \mathrm{a}\end{array}$ & $\begin{array}{c}(58.3) \\
17.4 \mathrm{ab}\end{array}$ & $\begin{array}{l}(24.2) \\
24.3 \mathrm{a}\end{array}$ & $\begin{array}{l}(34.7) \\
22.5 \mathrm{a}\end{array}$ \\
\hline $\begin{array}{c}40-20- \\
10\end{array}$ & 0.0 & Conv. & Agua & $\begin{array}{c}(28.4) \\
23.5 \mathrm{ab}\end{array}$ & $\begin{array}{c}(41.7) \\
24.3 \mathrm{ab}\end{array}$ & $\begin{array}{c}(31.2) \\
27.8 \mathrm{ab}\end{array}$ & $\begin{array}{c}(6.0) \\
21.5 \mathrm{a}\end{array}$ & $\begin{array}{c}(50.0) 18.3 \\
\mathrm{ab}\end{array}$ & $\begin{array}{c}(24.4) \\
24.0 \mathrm{ab}\end{array}$ & $\begin{array}{c}(33.0) \\
13.5 \mathrm{ab}\end{array}$ \\
\hline $\begin{array}{c}\text { 20-10- } \\
00\end{array}$ & 0.0 & Conv. & Agua & $\begin{array}{c}(29.0) \\
23.5 \mathrm{ab}\end{array}$ & $\begin{array}{l}(33.3) \\
18.7 \mathrm{~b}\end{array}$ & $\begin{array}{l}(34.1) \\
35.0 \mathrm{a}\end{array}$ & $\begin{array}{c}(5.5) \\
12.5 \mathrm{a}\end{array}$ & $\begin{array}{l}(27.8) \\
10.0 \mathrm{~b}\end{array}$ & $\begin{array}{c}(23.2) \\
19.5 \mathrm{ab}\end{array}$ & $\begin{array}{c}(33.0) \\
16.5 \mathrm{ab}\end{array}$ \\
\hline $\begin{array}{c}\text { 00-00- } \\
00\end{array}$ & 3.0 & Conv. & Agua & $\begin{array}{c}(26.7) \\
18.5 \mathrm{ab}\end{array}$ & $\begin{array}{c}(70.8) \\
27.1 \mathrm{ab}\end{array}$ & $\begin{array}{l}(27.2) \\
13.5 \mathrm{~b}\end{array}$ & $\begin{array}{c}(6.0) \\
22.1 \mathrm{a}\end{array}$ & $\begin{array}{c}(72.5) \\
19.9 \mathrm{ab}\end{array}$ & $\begin{array}{c}(22.5) \\
15.5 \mathrm{ab}\end{array}$ & $\begin{array}{c}(33.1) \\
17.0 \mathrm{ab}\end{array}$ \\
\hline $\begin{array}{c}\text { 00-00- } \\
00\end{array}$ & 1.0 & Conv. & Agua & $\begin{array}{c}(27.8) \\
17.3 \mathrm{ab}\end{array}$ & $\begin{array}{l}(83.3) \\
39.3 \mathrm{a}\end{array}$ & $\begin{array}{l}(29.0) \\
18.7 \mathrm{~b}\end{array}$ & $\begin{array}{c}(5.6) \\
13.0 \mathrm{a}\end{array}$ & $\begin{array}{l}(100) \\
31.5 \mathrm{a}\end{array}$ & $\begin{array}{c}(21.5) \\
15.3 \mathrm{ab}\end{array}$ & $\begin{array}{c}(33.4) \\
15.7 \mathrm{ab}\end{array}$ \\
\hline $\begin{array}{c}\text { 00-00- } \\
00\end{array}$ & 0.1 & Conv. & Agua & $\begin{array}{c}(29.0) \\
25.0 \mathrm{ab}\end{array}$ & $\begin{array}{l}(22.2) \\
11.5 \mathrm{~b}\end{array}$ & $\begin{array}{c}(35.1) \\
31.7 \mathrm{ab}\end{array}$ & $\begin{array}{c}(6.2) \\
21.3 \mathrm{a}\end{array}$ & $\begin{array}{l}(100) \\
31.5 \mathrm{a}\end{array}$ & $\begin{array}{l}(22.5) \\
17.3 \mathrm{ab}\end{array}$ & $\begin{array}{c}(35.0) \\
20.3 \mathrm{ab}\end{array}$ \\
\hline $\begin{array}{c}\text { 00-00- } \\
00\end{array}$ & 0.0 & Conv. & Agua & $\begin{array}{l}(30.2) \\
30.3 \mathrm{a}\end{array}$ & $\begin{array}{l}(20.8) \\
10.5 b\end{array}$ & $\begin{array}{l}(28.7) \\
19.8 \mathrm{~b}\end{array}$ & $\begin{array}{c}(6.5) \\
26.8 \mathrm{a}\end{array}$ & $\begin{array}{c}(50.0) \\
18.3 \mathrm{ab}\end{array}$ & $\begin{array}{l}(22.2) \\
17.5 \mathrm{ab}\end{array}$ & $\begin{array}{c}(35.5) \\
20.3 \mathrm{ab}\end{array}$ \\
\hline $\begin{array}{c}90-40- \\
20\end{array}$ & 0.0 & $\begin{array}{l}\text { Orgá- } \\
\text { nico }\end{array}$ & Agua & $\begin{array}{c}(26.7) \\
15.7 \mathrm{ab}\end{array}$ & $\begin{array}{c}(38.9) \\
21.0 \mathrm{ab}\end{array}$ & $\begin{array}{l}(26.9) \\
08.3 \mathrm{~b}\end{array}$ & $\begin{array}{l}(5.5) \\
10.0 \mathrm{a}\end{array}$ & $\begin{array}{l}(33.3) \\
13.8 \mathrm{~b}\end{array}$ & $\begin{array}{l}(19.3) \\
9.0 \mathrm{ab}\end{array}$ & $\begin{array}{l}(32.5) \\
3.0 \mathrm{~b}\end{array}$ \\
\hline $\begin{array}{c}90-40- \\
20\end{array}$ & 0.0 & Conv. & $10 \mathrm{CH}$ & $\begin{array}{l}(28.4) \\
21.9 \mathrm{ab}\end{array}$ & $\begin{array}{l}(33.3) \\
18.9 \mathrm{~b}\end{array}$ & $\begin{array}{l}(34.1) \\
36.0 \mathrm{a}\end{array}$ & $\begin{array}{r}(5.9) \\
15.5 \mathrm{a}\end{array}$ & $(100)$ & $\begin{array}{l}(22.4) \\
26.3 \mathrm{a}\end{array}$ & $\begin{array}{c}(34.2) \\
19.3 \mathrm{ab}\end{array}$ \\
\hline $\begin{array}{c}90-40- \\
20\end{array}$ & 0.0 & Conv. & $200 \mathrm{CH}$ & $\begin{array}{c}(28.5) \\
18.3 \mathrm{ab}\end{array}$ & $\begin{array}{c}(50.0) \\
27.8 \mathrm{ab}\end{array}$ & $\begin{array}{c}(29.4) \\
22.5 \mathrm{ab}\end{array}$ & $\begin{array}{c}(5.7) \\
12.8 \mathrm{a}\end{array}$ & $\begin{array}{l}(100) \\
31.5 \mathrm{a}\end{array}$ & $\begin{array}{c}(20.8) \\
16.0 \mathrm{ab}\end{array}$ & $\begin{array}{c}(34.2) \\
15.8 \mathrm{ab}\end{array}$ \\
\hline $\begin{array}{c}\text { 00-00- } \\
00\end{array}$ & 3.0 & $\begin{array}{l}\text { Orgá- } \\
\text { nico }\end{array}$ & Agua & $\begin{array}{l}(27.0) \\
14.7 \mathrm{ab}\end{array}$ & $\begin{array}{l}(33.3) \\
17.0 \mathrm{~b}\end{array}$ & $\begin{array}{l}(28.2) \\
12.3 \mathrm{~b}\end{array}$ & $\begin{array}{c}(6.2) \\
17.8 \mathrm{a}\end{array}$ & $(100)$ & $(18.6)$ & $\begin{array}{c}(33.2) \\
13.7 \mathrm{ab}\end{array}$ \\
\hline $\begin{array}{c}\text { 00-00- } \\
00\end{array}$ & 0.0 & Conv. & $200 \mathrm{CH}$ & $\begin{array}{l}(29.9) \\
32.0 \mathrm{a}\end{array}$ & $\begin{array}{c}(61.1) \\
31.8 \mathrm{ab}\end{array}$ & $\begin{array}{l}(28.7) \\
15.7 \mathrm{~b}\end{array}$ & $(5.7)$ & $\begin{array}{c}(68.3) \\
20.2 \mathrm{ab}\end{array}$ & $\begin{array}{l}(22.5) \\
17.7 \mathrm{ab}\end{array}$ & $(35.0)$ \\
\hline
\end{tabular}




\begin{tabular}{|c|c|c|c|c|c|c|c|c|c|c|}
\hline \multicolumn{2}{|c|}{ Fertilizante } & \multirow[b]{2}{*}{ Suelo } & \multirow[b]{2}{*}{$\begin{array}{l}\text { Agrono- } \\
\text { sode }\end{array}$} & \multirow[b]{2}{*}{$\begin{array}{c}\text { DL } \\
(\mathbf{d}) \\
\text { rango }^{*}\end{array}$} & \multirow{2}{*}{$\begin{array}{c}\text { VL } \\
\text { (por- } \\
\text { centaje) } \\
\text { rango }\end{array}$} & \multirow[b]{2}{*}{$\begin{array}{c}\text { PP } \\
\text { (mg) } \\
\text { rango }\end{array}$} & \multirow[b]{2}{*}{$\begin{array}{l}\text { DP } \\
\text { (d) } \\
\text { rango }\end{array}$} & \multirow{2}{*}{$\begin{array}{c}\text { VP } \\
\begin{array}{c}\text { (por- } \\
\text { centaje) } \\
\text { rango }\end{array}\end{array}$} & \multirow[b]{2}{*}{$\begin{array}{c}\text { PA } \\
\text { (mg) } \\
\text { rango }\end{array}$} & \multirow[b]{2}{*}{$\begin{array}{l}\text { DLA } \\
\text { (d) } \\
\text { rango }\end{array}$} \\
\hline $\begin{array}{l}\text { Mine- } \\
\text { ral } \\
(\mathrm{NPK})\end{array}$ & $\begin{array}{l}\text { Com- } \\
\text { posta } \\
\left(\mathrm{t} \mathrm{ha}^{-1}\right)\end{array}$ & & & & & & & & & \\
\hline $\begin{array}{c}\text { 00-00- } \\
00\end{array}$ & 0.0 & $\begin{array}{c}\text { Orgá- } \\
\text { nico }\end{array}$ & $200 \mathrm{CH}$ & $\begin{array}{r}(27.8) \\
25.8 \mathrm{ab}\end{array}$ & $\begin{array}{l}(27.8) \\
14.7 \mathrm{~b}\end{array}$ & $\begin{array}{r}(31.7) \\
30.2 \mathrm{ab}\end{array}$ & $(8.0)^{\mathrm{NA}}$ & $(14.3)^{\mathrm{NA}}$ & $(08.5)^{\mathrm{NA}}$ & $(35.8)^{\mathrm{NA}}$ \\
\hline $\begin{array}{c}\text { 00-00- } \\
00\end{array}$ & 0.0 & $\begin{array}{l}\text { Orgá- } \\
\text { nico }\end{array}$ & Agua & $\begin{array}{l}(25.8) \\
03.5 \mathrm{~b}\end{array}$ & $\begin{array}{r}(41.7) \\
24.3 \mathrm{ab}\end{array}$ & $\begin{array}{l}(28.0) \\
13.8 \mathrm{~b}\end{array}$ & $\begin{array}{c}(5.5) \\
12.5 \mathrm{a}\end{array}$ & $\begin{array}{l}(58.3) \\
15.0 \mathrm{~b}\end{array}$ & $\begin{array}{r}(23.0) \\
20.5 \mathrm{ab}\end{array}$ & $\begin{array}{l}(31.5) \\
4.3 \mathrm{~b}\end{array}$ \\
\hline
\end{tabular}

* Medias de rangos con letras similares en columnas no son diferentes estadísticamente de acuerdo con la prueba de Tukey $(\alpha \leq 0.05)$. NA: no analizado debido a falta de datos suficientes para el análisis estadístico.

La DL fue menor significativamente en suelo orgánico sin fertilización y agronosode; la VL se incrementó significativamente con el tratamiento de abono orgánico $1 \mathrm{t} \mathrm{ha}^{-1} \mathrm{y}$ en $\mathrm{PP}$ se observó un incremento significativo en los tratamientos de fertilizante mineral en dosis baja y el agronosode a la 10CH. La reducción de la duración larval, mencionada anteriormente como efecto de la mala alimentación de la larva por la aplicación de composta, también se provoca en suelo orgánico como ahora se estipula; incluso puede darse con alta dosis de abono orgánico como lo señala Boiteau et al. (2008). En contraste, la duración larval se puede incrementar con el abono orgánico como lo mencionan Hsu et al. (2009) con la mariposita blanca de la col (P. rapae crucivora) y Johnson et al. (2012) con la palomilla dorso de diamante (P. xylostella).

Con base en lo encontrado en este segundo experimento se infiere que el suelo orgánico afecta la DL, reduciéndola en 4.4 días, y en el caso del agronosode a la $200 \mathrm{CH}$, que no tiene efecto por sí mismo, combinado con el suelo orgánico, que acorta la $\mathrm{DL}$, afecta de manera inusitada la supervivencia de las pupas; de forma tal que, emerge solo una séptima parte de adultos de la población tratada desde primer instar.

El efecto del suelo orgánico en el insecto ya había sido reportado por Phelan et al. (1995), quienes mencionaron que el suelo orgánico disminuyó la preferencia para oviposición del barrenador europeo del maíz O. nubilalis.

De manera general, en esta investigación se observó que la fertilización, los suelos y los agronosodes influyen en la biología de la conchuela del frijol y en el daño diario del adulto; fertilizar con composta o sembrar en suelo orgánico reduce la duración larval y combinar éste último con agronosode a la $200 \mathrm{CH}$ disminuye la supervivencia pupal de 
la conchuela del frijol E. varivestis. Además, la composta y el agronosode a la $200 \mathrm{CH}$, por separado, protegen al cultivo, siendo menor el efecto con el homeopático. Al respecto Marshner (1995), menciona que en un suelo con mayor fertilidad se estimulan los mecanismos de defensa de la planta y se reduce la herbivoría, y Phelan et al. (1996) añaden que la fertilidad tiene un efecto de disminución de la preferencia del insecto a largo plazo por el equilibrio mineral que se establece en ese suelo.

Por otro lado, Ronquillo et al. (2013), también encontraron resultados similares, puesto que aducen menor duración larval y peso de adulto con la aplicación de composta respecto al fertilizante mineral nitrogenado. Por su parte, Johnson et al. (2012), encontraron menor peso y supervivencia de P. xylostella con la aplicación de abono orgánico. Esto indica las mejoras obtenidas con este manejo, dado que además, la aplicación de composta reduce el daño del adulto de conchuela, alimentado de las mismas plantas en las que se desarrolló como larva.

En el caso de la agrohomeopatía, este trabajo que incluye el desarrollo del insecto y el daño al cultivo, muestra que si bien no hay un efecto importante en los parámetros biológicos, aunque la combinación de suelo orgánico y agronosode a la $200 \mathrm{CH}$ si lo tiene a partir de la etapa de pupa, existe una protección al cul- tivo, por la reducción significativa de daño, respecto a la fertilización mineral, lo que lo hace interesante, ya que las investigaciones publicadas se han enfocado únicamente en el daño de la plaga o desarrollo del insecto, y los pocos estudios en ambos factores no muestran efecto, como Ramírez et al. (2014), que al aplicar agronosodes a la 6, 10 y $30 \mathrm{CH}$ en frijol no redujeron el consumo ni peso de la larva de conchuela del frijol E. varivestis.

Por otra parte, la reducción del daño diario del adulto de la conchuela con el agronosode $200 \mathrm{CH}$, coincide con lo observado por Giesel et al. (2012 y 2013), quienes constataron menor actividad de forrajeo de las hormigas defoliadoras de Acromyrmex sp. $y$ de A. sexdens piriventris con el agronosode a la $30 \mathrm{CH}$.

Sin embargo, es necesario continuar con la investigación realizando trabajos donde se incluya la interacción de la fertilización y los agronosodes con factores del suelo, ya que cuando se combinó el agronosode a la $200 \mathrm{CH}$ con composta $3 \mathrm{t} \mathrm{ha}^{-1}$ se perdió el efecto de reducción del daño que ambos tuvieron como tratamientos simples.

Por tal razón, la aportación de este trabajo radica en la posibilidad de proteger los cultivos, induciendo resistencia en el frijol, a través de la fertilización orgánica, por tiempos prolongados para incrementar la fertilidad del suelo y hacer uso del agronosode a la $200 \mathrm{CH}$, como parte de un manejo agroecológico de plagas; 
el cual es acorde con los principios de la agricultura orgánica, ya que respeta los procesos biogeoquímicos y hace uso de los recursos locales en la fabricación de los insumos utilizados. Esto brinda la oportunidad de obtener un producto sano, inocuo y de calidad que beneficia al productor por el menor gasto en estrategias de control y al consumidor por las características de calidad mencionadas.

\section{Conclusiones}

La alimentación de la larva de la conchuela del frijol E. varivestis en frijol cultivado con fertilización mineral u orgánica, suelo convencional u orgánico, y sin o con agronosode afecta el ciclo biológico y el daño del adulto. La fertilización orgánica (composta) 1 y $3 \mathrm{t}$ $\mathrm{ha}^{-1} \mathrm{y}$ el suelo orgánico, por separado, reducen de 7 a $14.6 \%$ la duración larval, respecto a la fertilización mineral y al suelo convencional.
La aplicación de composta en dosis de 1.8 a $3.6 \mathrm{t} \mathrm{ha}^{-1} \mathrm{y}$ del agronosode a la $200 \mathrm{CH}$ reduce en más de 50 y en 50\% el daño diario del adulto, respectivamente; y cuando se combinan se pierde el efecto. El suelo orgánico combinado con la aplicación de agronosode a la $200 \mathrm{CH}$ vía riego disminuye en $85.7 \%$ la supervivencia de la pupa de la conchuela.

Para la realización de esta investigación se agradece de forma especial al Consejo $\mathrm{Na}$ cional de ciencia y Tecnología (CONACyT) de México por la beca otorgada, así como al Colegio de Postgraduados en ciencias agrícolas por la oportunidad. A la Dra. María de la Nieves, por facilitar el espacio donde se inició el trabajo de campo; y al Dr. Moisés Cuevas Vázquez por facilitar el espacio donde finalmente se realizó el primer experimento y la composta utilizada en el estudio

\section{Referencias}

Boiteau, G.; Lynch y Martin, R.C. (2008). Influence of fertilization on the colorado potato beetle, Leptinotarsa decemlineata, in organic potato production. Environmental Entomo$\log y 37(2)$, pp. 575-585.

Conover, W.J. y Iman, R.L. (1981). Rank transformations as a bridge between parametric and nonparametric statistics. The American Statistician 35(3), pp. 124-129.

Deboni, T.C. (2009). Preparados homeopáticos e fitoterápicos no manejo de Acanthoscelides obtectus Say, 1831 (Coleoptera: Bruchidae) em feijão armazenado. Tesis de maestría no publicada. Universidade do Estado de Santa Catarina: Brasil. Recuperado de: http:// tede.udesc.br/bitstream/handle/1118/1/PGPV09MA072.pdf 
Dixit, A.; Dixit, L. y Samson, M. (2009). Effect of soil fertilization of feeding behavior and development of last instar nymphs of grasshopper, Hieroglyphus nigrorepletus Bolivar. Karnataka Journal of Agricultural Sciences 22(3-spl. issue), pp. 644-645.

Giesel, A.; Boff, M. I. C. y Boff, P. (2012). The effect of homeopathic preparations on the activity level of Acromyrmex leaf-cutting ants. Acta Scientiarum. Agronomy 34(4), pp. 445-451.

Giesel, A.; Boff, M.I.C.; Gonçalves, P.A.D.S. y Boff, P. (2013). Activity of leaf-cutting ant Atta sexdens piriventris submited to high dilution homeopathic preparations. Tropical and Subtropical Agroecosystems 16(1), pp. 25-33.

Hsu, Y.T.; Shen, T.C. y Hwang, S.Y. (2009). Soil fertility management and pest responses: a comparison of organic and synthetic fertilization. Journal of Economic Entomology 102(1), pp. 160-169.

Johnson, W.A.; Cloyd, R.A.; Nechols, J.R.; Williams, K.A.; Nelson, N.O.; Rotenberg, D. y Kennelly, M.M. (2012). Effect of nitrogen source on pac choi (Brassica rapa L.) chemistry and interactions with the diamondback moth (Plutella xylostella L.). HortScience 47(10), pp. 1457-1465.

Marshner, H. (1995). Mineral Nutrition of Higher Plants. New York, USA: Academic Press.

Phelan, P.L.; Mason, J.F. y Stinner, B.R. (1995). Soil-fertility management and host preference by european corn borer, Ostrinia nubilalis (Hübner), on Zea mays L.: a comparison of organic and conventional chemical farming. Agriculture, Ecosystems and Environment 56(1), pp. 1-8.

Phelan, P.L.; Norris, K.H. y Mason, J.F. (1996). Soil-management history and host preference by Ostrinia nubilalis: evidence for plant mineral balance mediating insect-plant interactions. Environmental Entomology 25(6), pp. 1329-1336.

Ramírez, O., S.M.; Rodríguez, H.C.; Curiel, R. A. y Ruiz, E.F.J. (2014). Tratamientos homeopáticos para disminuir la defoliación de conchuela Epilachna varivestis (Coleoptera: Coccinellidae) a frijol. In: Ruiz E., F.J.; Betancourt V. J.G., y Tinajero A. S. (Coords.). Homeopatía: Avances 2014. Universidad Autónoma Chapingo. Chapingo, Texcoco, Estado de México, México, pp. 77-84. 
Rasband, W.S. (2014). ImageJ Versión 1.48. National Institutes of Health, Bethesda, Maryland, USA. Recuperado de http://imagej.nih.gov/ij/.

Ronquillo, C.I.; Rodríguez, H. C.; Curiel, R. A., y Cuevas, V M. (2013). Actividad antialimentaria contra la conchuela del frijol Epilachna varivestis (Coleoptera: Coccinellidae) en hojas de frijol cultivado con abono orgánico. In: Álvarez G., F.; Ortiz T. E.; Bahena J.F.; Pérez M. A.; Carranza C. I.; Pérez R. E.; Díaz R. R.; Villanueva J. J.A.; Ocampo F I.., y Villarreal M.L.A.. (eds). Agricultura Sostenible Vol. 9. Sociedad Mexicana de Agricultura Sostenible. Puebla, México. pp. 2828-2836.

Ruiz, E.F.J. y Rodríguez, H.C. (2013). La agrohomeopatía en el manejo de plagas agropecuarias. In: Rodríguez H. C., y Guzmán M.R. (eds). Métodos Bioracionales para el Manejo de Plagas. Agricultura sostenible 8. Colegio de Postgraduados y Sociedad Mexicana de Agricultura Sostenible. Montecillo, Texcoco, Estado de México, México, pp.91-99.

Rupp, L.C.D.; Boff, M.I.C.; Boff, P.; Gonçalves, P.A.S. y Botton, M. (2012). High dilution of Staphysagria and fruit fly biotherapic preparations to manage South American fruit fly, Anastrepha fraterculus, in organic peach orchards. Biological Agriculture and Horticulture 28(1), pp. 41-48.

SAS Institute. (2004). SAS/STAT ${ }^{\oplus}$ Version 9.1. SAS Institute Inc. Cary, NC, USA.

Wyss, E; Tamm, L.; Siebenwirth, J. y Baumgartner, S. (2010). Homeopathic preparations to control the rosy apple aphid (Dysaphis plantaginea Pass.). The scientific world journal 10, pp. 38-48.

Yardim, E.N.; Arancon, N.Q.; Edwars, C.A.; Oliver, T.J. y Byrne R.J. (2006). Suppression of tomato hornworm (Manduca quinquemaculata) and cucumber beetles (Acalymma vittatum and Diabrotica undecimpunctata) populations and damage by vermicompost. Pedobiologia 50(1), pp. 23-29 